\section{RMD Open}

Rheumatic \&

Musculoskeletal Diseases

\title{
Metabolomic profiling predicts outcome of rituximab therapy in rheumatoid arthritis
}

\author{
Shannon R Sweeney, ${ }^{1}$ Arthur Kavanaugh, ${ }^{2}$ Alessia Lodi, ${ }^{1}$ Bo Wang, ${ }^{1}$ David Boyle, ${ }^{2}$ \\ Stefano Tiziani, ${ }^{1}$ Monica Guma ${ }^{2}$
}

To cite: Sweeney SR, Kavanaugh A, Lodi A, et al. Metabolomic profiling predicts outcome of rituximab therapy in rheumatoid arthritis. $R M D$ Open 2016;2:e000289. doi:10.1136/rmdopen-2016000289

- Prepublication history and additional material is available. To view please visit the journal (http://dx.doi.org/ 10.1136/rmdopen-2016000289).

ST and MG contributed equally.

Received 1 April 2016

Revised 27 June 2016 Accepted 21 July 2016

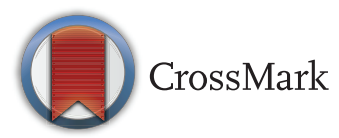

${ }^{1}$ Department of Nutritional Sciences, Dell Pediatric Research Institute, University of Texas at Austin, Austin, Texas, USA

${ }^{2}$ Division of Rheumatology, Allergy and Immunology, UC San Diego School of Medicine, La Jolla, California, USA

Correspondence to Stefano Tiziani; tiziani@ austin.utexas.edu and Monica Guma; mguma@ucsd.edu

\section{ABSTRACT}

Objective: To determine whether characterisation of patients' metabolic profiles, utilising nuclear magnetic resonance (NMR) and mass spectrometry (MS), could predict response to rituximab therapy. 23 patients with active, seropositive rheumatoid arthritis (RA) on concomitant methotrexate were treated with rituximab. Patients were grouped into responders and nonresponders according to the American College of Rheumatology improvement criteria, at a $20 \%$ level at 6 months. A Bruker Avance $700 \mathrm{MHz}$ spectrometer and a Thermo Scientific $Q$ Exactive Hybrid QuadrupoleOrbitrap mass spectrometer were used to acquire ${ }^{1} \mathrm{H}-\mathrm{NMR}$ and ultra high pressure liquid chromatography (UPLC)-MS/MS spectra, respectively, of serum samples before and after rituximab therapy. Data processing and statistical analysis were performed in MATLAB. 14 patients were characterised as responders, and 9 patients were considered nonresponders. 7 polar metabolites (phenylalanine, 2-hydroxyvalerate, succinate, choline, glycine, acetoacetate and tyrosine) and 15 lipid species were different between responders and non-responders at baseline. Phosphatidylethanolamines,

phosphatidyserines and phosphatidylglycerols were downregulated in responders. An opposite trend was observed in phosphatidylinositols. At 6 months, 5 polar metabolites (succinate, taurine, lactate, pyruvate and aspartate) and 37 lipids were different between groups. The relationship between serum metabolic profiles and clinical response to rituximab suggests that ${ }^{1} \mathrm{H}$-NMR and UPLC-MS/MS may be promising tools for predicting response to rituximab.

\section{INTRODUCTION}

Early detection and initiation of an effective treatment in rheumatoid arthritis (RA) is critical for minimising damage caused by the disease and improving immediate and longterm patient outcomes and quality of life. Aggressive treatment is key if the damage caused by RA is to be controlled. In particular, successful disease management requires

\section{Key messages}

What is already known about this subject?

- Current methods make it challenging to accurately predict rituximab response in patients with rheumatoid arthritis.

What does this study add?

- This study demonstrates differential metabolism between patients who respond to rituximab and those who do not and identifies several metabolites and pathways as potential biomarkers.

How might this impact on clinical practice?

- Metabolite profiles can differentiate rituximab responders and non-responders when other clinical measures fail to do so, thus streamlining treatment protocols.

better tools for diagnosis and streamlining of treatment protocols. ${ }^{12}$ Thus, if choosing and initiating the right biological treatment earlier in the course of disease could help to reach the goal of remission, a greater effort should be made to develop the tools necessary to employ a 'personalised' medicine approach, in an attempt to match patients with the most appropriate therapy option for their disease subtype.

Once genetic and epigenetic risk factors and environmental triggers have led from preclinical to clinical disease, RA may be driven by several different factors, including cytokines, such as tumour necrosis factor (TNF) or interleukin 6 (IL-6), or different cell subset, such as B cell, T cell or macrophages, which ultimately lead the perpetuating cycle of chronic synovitis. ${ }^{3} 4$ Given the complexity and heterogeneity of RA, it seems doubtful that a single cytokine or biomarker will be sufficient for therapy discrimination. Instead, biomarker signatures may represent more realistic approach for the future of 
personalised therapeutic protocols for those suffering from the disease. ${ }^{5}$ Identifying these unique signatures could make a significant difference in RA management and attainment of disease remission. Metabolomics is the science of identifying and quantifying the biochemical by-products of metabolism, frequently referred to as metabolites. ${ }^{67}$ The goal of metabolomics is to comprehensively measure the small molecules present in a specific cell, tissue, organ, organism or biofluids. ${ }^{6-8}$ Variations in metabolite concentrations can serve as diagnostic or prognostic biomarkers. We propose that the study of metabolomics in RA can be useful to identify biomarker signatures. $^{9-11}$ Metabolomics has many applications and is frequently used to identify single biomarkers, classify metabolite patterns of health or disease, elucidate pathways involved in pathogenesis, uncover novel targets for modulation of dysregulated pathways and to monitor treatment and/or disease status. ${ }^{12-14}$ Recent studies in other fields, such as oncology, demonstrate the applicability of metabolomics using serum and urine samples for diagnosis and prognosis. ${ }^{15-21}$ The application of metabolomics to RA is still in its infancy, but early studies have yielded promising results. ${ }^{22-27}$ These studies suggest that metabolomics analyses of several different biological fluids may be useful diagnostic tools prior to initiation of treatment and may also prove effective for earlier detection of RA. They also suggest that metabolic profiling has the potential to effectively predict patient response to therapy prior to administration. Here, we show that an untargeted analysis of polar and lipid metabolites from serum samples is a promising clinical tool for predicting response to rituximab therapy and ultimately improving patient outcomes.

\section{METHODS}

\section{Patients and clinical outcomes}

The ARISE (Assessment of Rituximab's Immunomodulatory Synovial Effects registered at ClinicalTrials.Gov NCT00147966) clinical trial was recently described in detail $^{28}$ and is briefly described in online supplementary material. The primary clinical outcome was response according to the ACR improvement criteria, at a $20 \%$ level (ACR20), at 6 months. Patients who left the study before 6 months were considered non-responders. Secondary clinical outcomes included ACR20 as well as ACR50 and ACR70 responses at monthly time points, Disease Activity Score using a 28-joint count (DAS28) ${ }^{29}$ and changes in individual disease activity parameters: tender joint count, swollen joint count, physician global assessment of disease, patient global assessment of disease, patient assessment of pain, measure of functional status using the Health Assessment Questionnaire (HAQ) and Creactive protein (CRP) and erythrocyte sedimentation rate (ESR) at monthly time points.

\section{Metabolomics analysis}

A total of 43 samples were prepared and analysed using nuclear magnetic resonance (NMR) and ultra high pressure liquid chromatography (UPLC)-mass spectrometry (MS)/MS analytical platforms ${ }^{10} \quad 15 \quad 30-33$ as described in online supplementary material. Frozen sera were obtained from the Division of Rheumatology, Allergy and Immunology at UC San Diego School of Medicine (San Diego, California, USA) for polar and lipid analyses. NMR spectra were acquired with a $16.4 \mathrm{~T}$ $(700 \mathrm{MHz})$ Bruker Avance spectrometer (Bruker BioSpin, Billerica, Massachusetts, USA) equipped with a $5 \mathrm{~mm}$ TCI cryogenically cooled probe and an autosampler at $30^{\circ} \mathrm{C}$. Following acquisition, spectra were processed using NMRlab and MetaboLab. ${ }^{34}$ Metabolite assignment and quantification were performed using several database. ${ }^{3135}$

UPLC-MS analysis on lipid fraction was performed on a Q Exactive Hybrid Quadrupole-Orbitrap Mass Spectrometer equipped with an Accela 1250 pump and an autosampler as described in online supplementary material (Thermo Scientific, Waltham, Massachusetts, USA). Metabolite assignment was performed at a $5 \mathrm{ppm}$ mass accuracy range by interrogation of several databases. $^{35-40}$ MetaboAnalyst V.3.0 $0^{41}$ and VANTED ${ }^{42}$ software were used for metabolic pathway analysis (see online supplementary material).

\section{Statistical analysis}

Partial least squares discriminant analysis (PLSDA) with venetian blinds cross-validation was performed using PLS-Toolbox (Eigenvector Research, Manson, Washington, USA). Polar metabolite correlation relationships were reported in Pearson's correlation coefficients and visualised as a heat map with hierarchical clustering analysis with Euclidean distance metric by MATLAB. Statistical significance analysis between responders and non-responders was performed using an unpaired Student's t-test (statistical significance: $* \mathrm{p}<0.1, * * \mathrm{p}<0.05$ and $* * * \mathrm{p}<0.01)$.

\section{RESULTS}

\section{Patient characteristics}

After 6 months of rituximab therapy, patients were divided into two groups according to their response, as determined by the 1987 ACR classification criteria. At baseline, 14 patients were classified as responders and 9 as non-responders. ${ }^{28}$ Similarly, of the 20 patients who followed up at 6 months, 13 were responders and the remaining 7 were non-responders. With the exception of ACR response, no significant differences were observed between rituximab responders and non-responders at baseline. Age, gender, baseline methotrexate doses, nonsteroidal anti-inflammatory drugs (NSAIDs) and prednisone users were all comparable between groups. In addition, clinical disease parameters, including DAS, HAQ, pain, joint swelling and tenderness, ESR, rheumatoid factor (RF), anti-CCP and CD19+ cells were similar between groups prior to rituximab administration (see online supplementary table $\mathrm{S} 1$ ). 


\section{Metabolomics analysis}

Polar and apolar fractions were prepared for all 43 samples and analysed by $1 \mathrm{D}{ }^{1} \mathrm{H}-\mathrm{NMR}$ and UPLC-MS/ MS, respectively. ${ }^{31} \quad 35$ 38-42 Identified polar metabolites include amino acids, ketone bodies and intermediates of energy metabolism (see online supplementary table S2). Interrogation of several reference libraries yielded 584 known lipid compounds from the apolar fraction. ${ }^{35}$ 37-39 Several major classes of lipids were well represented including phosphatidic acids (PA), phosphatidylcholines (PC), phosphatidylethanolamines (PE), phosphatidylglycerols (PG), phosphatidylinositols (PI), phosphatidylserines (PS), sphingomyelins, ceramides, sterols, triglycerides and free fatty acids. Conventional ester, alkyl ether (O-prefix) and alkenyl ether (P-prefix) linkage glycerophospholipids were identified.

\section{Differences in metabolite profiles before treatment}

Polar metabolite profiles of patient sera before rituximab therapy was accomplished using $1 \mathrm{D}{ }^{1} \mathrm{H}-\mathrm{NMR}$ spectroscopy (figure $1 \mathrm{~A}(\mathrm{a})$ ). Thirteen polar metabolites had $\mathrm{p}<0.10$, seven of which achieved $\mathrm{p}<0.05$ (phenylalanine, 2-hydroxyvalerate, succinate, choline, glycine, acetoacetate and tyrosine; figure $1 \mathrm{~B}(\mathrm{a}))$. Mean spectra (figure $1 \mathrm{~A}(\mathrm{a})$ ) show that most, but not all features are diminished in responders. Pearson's correlation coefficients show positive relationships between identified polar metabolites. Metabolites primarily cluster into groups according to their biological function or chemical classification (see online supplementary figure $\mathrm{S} 1$ ).

Apolar metabolites were isolated by UPLC-MS/MS in negative and positive modes for the most complete lipidome coverage. Thousands of features were detected in the MS spectra, of which, 584 were matched to known compounds reported in established libraries. Forty-one identified compounds achieved $\mathrm{p}<0.10$. Of these lipids, 15 reached $\mathrm{p}<0.05$ (see online supplementary table S3). Of the statistically different lipids, $80 \%(12 / 15)$ were glycerophospholipids, suggesting that regulation and metabolism of this lipid class is an important difference between groups. Lipid species that were identified as statistically significant were grouped according to their classes. This revealed interesting trends among lipid species. Relative to non-responders, responders displayed elevated levels of ester-linked PI and PC. In
Figure 1 (A) Mean NMR spectra of patient sera show differences in metabolite intensities between responders and non-responders before (a) and 6 months after (b) treatment with rituximab. Variations in metabolite concentrations between groups indicate unique metabolite profiles between responders and non-responders at both time points. Labelled peaks indicate significant metabolites. (B) Concentrations of specific polar metabolites are different between rituximab responders and non-responders. The mean concentrations $( \pm S D)$ of sera polar metabolites of patients with RA measured by ${ }^{1} \mathrm{H}$-NMR before (a) and after (b) treatment with rituximab (lactate excluded due to scale). These metabolites represent a group of potential biomarkers for predicting and assessing patient response to rituximab treatment. ${ }^{*} \mathrm{p}<0.1$ ${ }^{\star *} p<0.05$. AcAc, acetoacetate; Glu, glutamate; NMR, nuclear magnetic resonance; RA, rheumatoid arthritis.
A

(a)

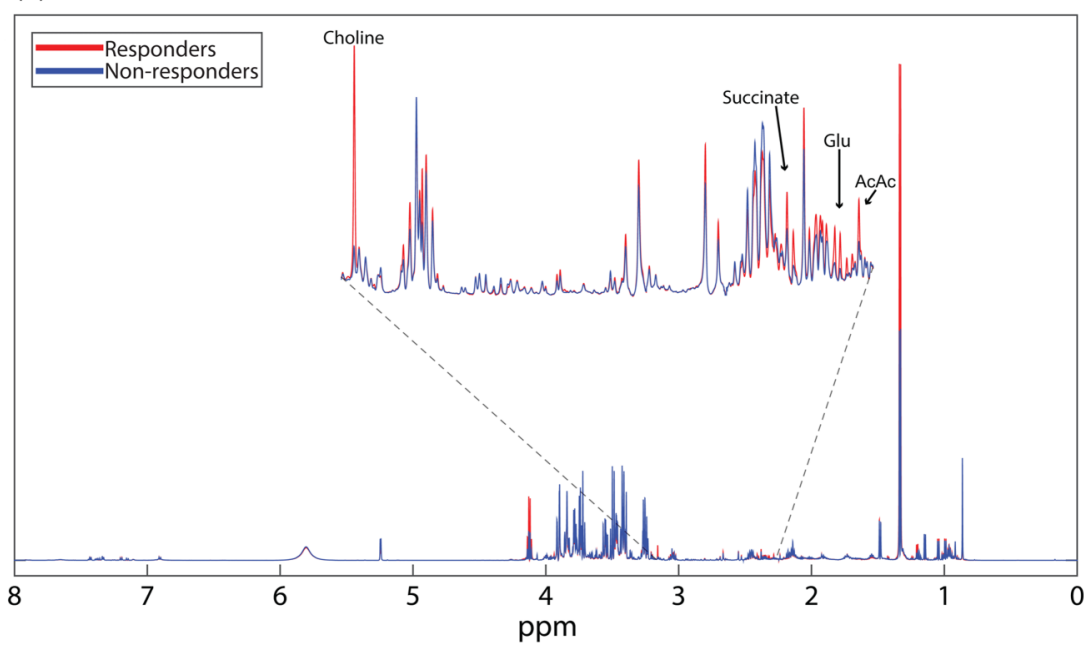

(b)

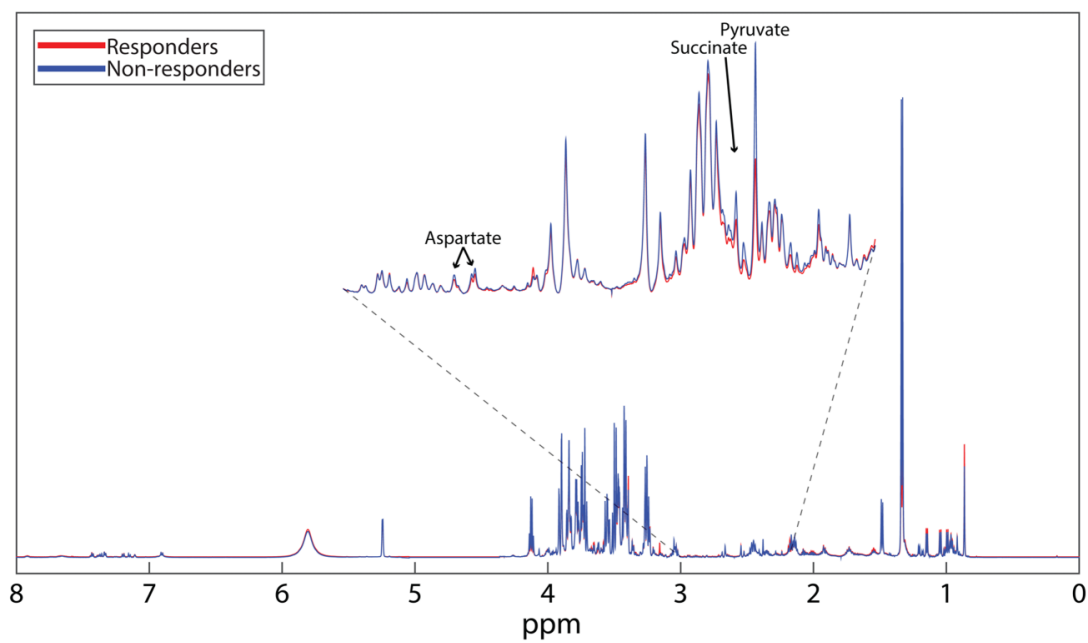


Figure 1 Continued

B

(a) 1.2

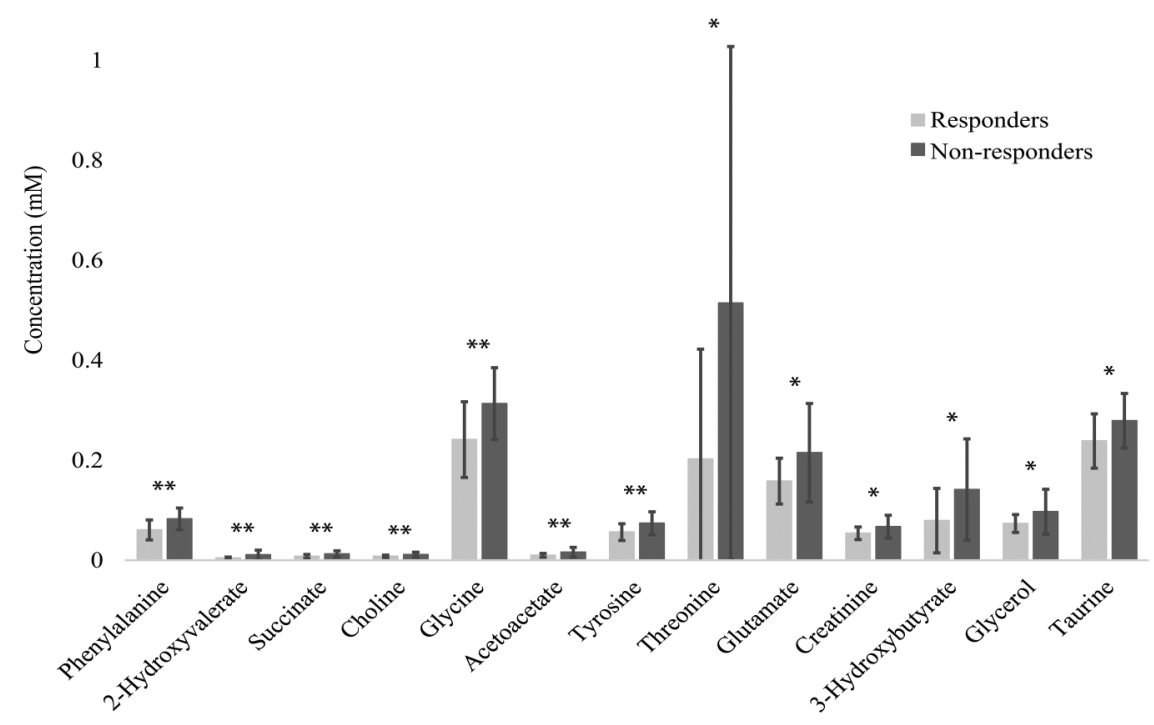

(b) 0.18

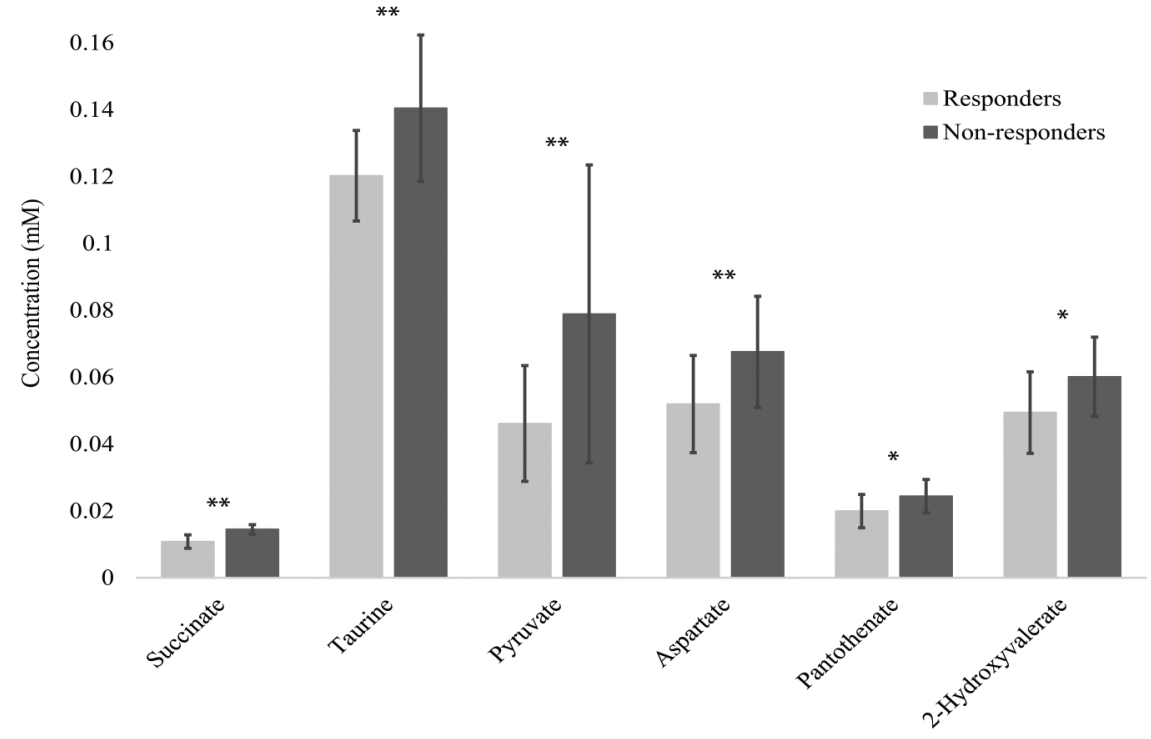

contrast, ester linked PG, all PE, all PS, O/P-PC and O/ $\mathrm{P}$-PI were lower in responders than non-responders (see online supplementary table S5).

\section{Differences in metabolite profiles following treatment}

Serum collected at the 6-month follow-up was used for metabolomics analysis. Mean 1D ${ }^{1} \mathrm{H}-\mathrm{NMR}$ spectra at 6 months (figure $1 \mathrm{~A}(\mathrm{~b})$ ) show clear differences between time points that are indicative of differential metabolic response to rituximab as well as the effects of rituximab on both populations despite clinical parameters. Polar metabolite quantification at 6 months revealed seven metabolites that attained $\mathrm{p}<0.10$ between responders and non-responders. Five of these metabolites, succinate, taurine, lactate, pyruvate and aspartate achieved $\mathrm{p}<0.05$ (figure $1 \mathrm{~B}(\mathrm{~b})$ ).
Following rituximab therapy, the composite UPLCMS analysis of the apolar fraction of patient sera resulted in the identification of 69 lipid species that achieved $\mathrm{p}<0.10$. Of these, 37 metabolites resulted in $\mathrm{p}<0.05$ (see online supplementary table S4). Similar to baseline, many of these lipid species belong to the glycerophospholipid class (24/37 or 65\%). Again, significant lipids were clustered by class to identify trends. Relative fold-changes were calculated for lipid species that were significant between responders and nonresponders (data not shown). Six months after treatment with rituximab, elevated levels of PA, PC, PS and $\mathrm{O} / \mathrm{P}-\mathrm{PG}$ were observed in responders relative to nonresponders. Conversely, PE, PG, O/P-PI, O/P-PC and $\mathrm{O} / \mathrm{P}-\mathrm{PS}$ were lower in responders than in nonresponders (table 1 ). 
Table 1 Pathway analysis of polar compounds by MetaboAnalyst

\begin{tabular}{lllll}
\hline Pathway & Total compounds & Hits & p Value (before) & p Value (after) \\
\hline Glycine, serine and threonine metabolism & 48 & 8 & 0.05111 & 0.0983 \\
Citrate cycle (TCA cycle) & 20 & 4 & 0.2849 & 0.0283 \\
Pyruvate metabolism & 32 & 3 & 0.7025 & 0.0218
\end{tabular}

Pathway $p$ values are calculated based on differences in metabolite concentrations between responders and non-responders before and after rituximab treatment.

TCA, tricarboxylic acid.
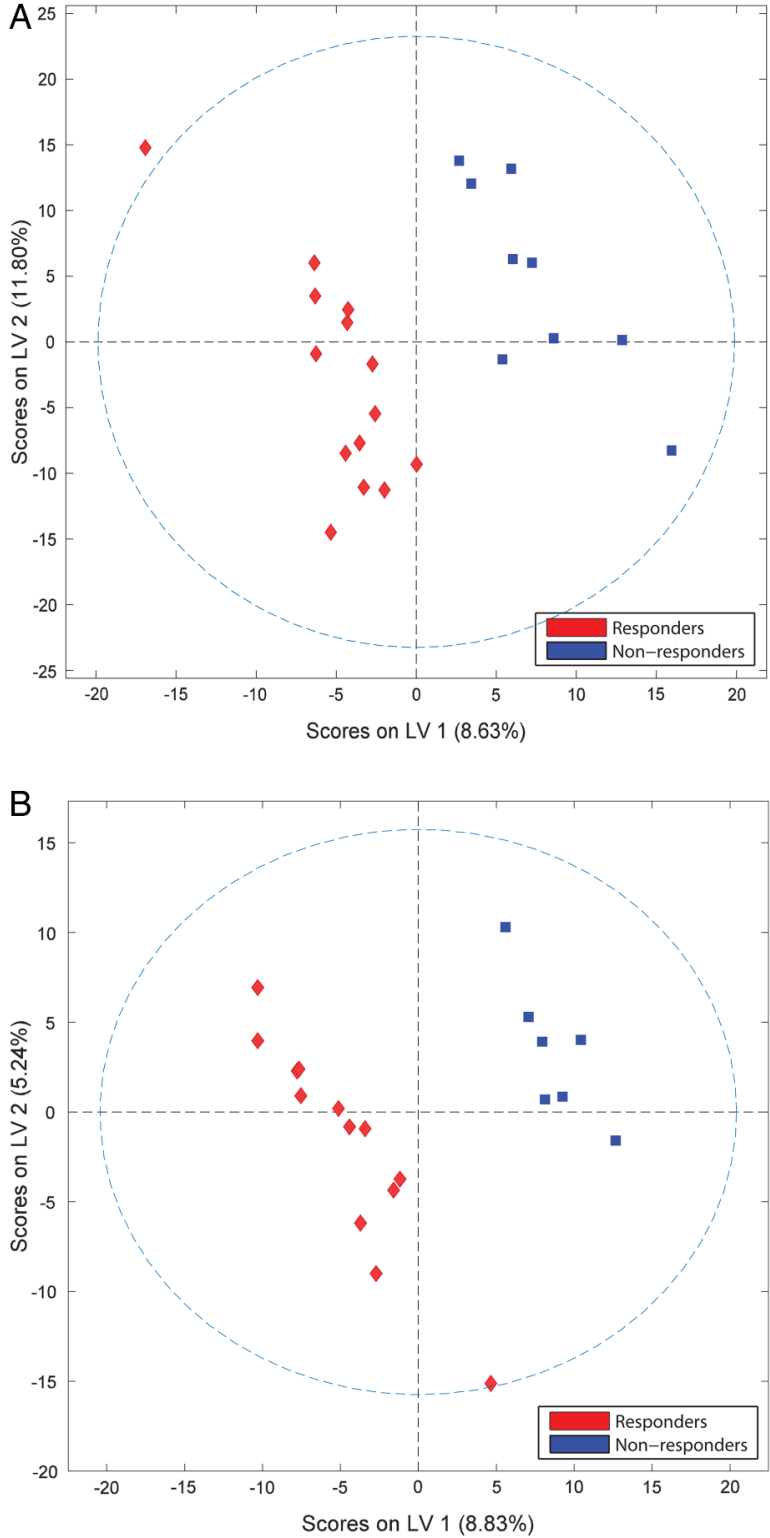

Figure 2 Sera metabolite profiles of patients with RA discriminate rituximab responders and non-responders. PLSDA score plots obtained from NMR and MS of samples collected before $(A)$ and after $(B)$ treatment with rituximab demonstrate group separation based on latent variables (LVs). LV1, latent variable 1; LV2, latent variable 2; NMR, nuclear magnetic resonance; MS, mass spectrometry; PLSDA, partial least squares discriminant analysis; RA, rheumatoid arthritis.

\section{Statistical analysis of metabolite profiles}

Polar and apolar data sets were combined to capture the relative contribution of each metabolite to the overall differences observed between responders and nonresponders before and after rituximab treatment. At both time points, PLSDA successfully separated responders and non-responders (figure 2). Pretreatment and post-treatment metabolites show similar degrees of separation, particularly with respect to latent variable 1 (LV1). Following treatment, separation is largely diminished in regard to latent variable 2 (LV2), reflecting the observed shifts in the metabolite profiles of both groups over time. Despite the relatively small latent variables, clear separation between groups is observed in both score plots, particularly in the $x$-direction. The $R^{2}$ values for pretreatment and post-treatment analyses were 0.91 and 0.97 , respectively (see online supplementary table S6).

Owing to the large number of apolar metabolites identified, lipids were grouped into general classes, to simplify analysis and draw conclusions based on metabolite chemistry and biological function as opposed to chain length or minor differences in double bond number and location. Fold changes were calculated using only the metabolites present in $>50 \%$ of the patient samples to ensure more robust results. This information was then used to generate a pathway to visualise global shifts in metabolite profiles as opposed to specific changes to individual metabolites.

\section{Pathway analysis}

Polar metabolites were mapped to known metabolic pathways using MetaboAnalyst V.3.0. ${ }^{41}$ Pathways were ranked by their overall $\mathrm{p}$ values (table 1). VANTED software ${ }^{42}$ was used to construct a metabolic pathway for glycerophospholipids, which represents the majority of the significant apolar metabolites identified at both time points ( $\geq 65 \%$ for both). Mixed polar and apolar pathway analysis were combined to provide a comprehensive picture of the global differences in metabolism between rituximab responders and non-responders (figure 3). Pathway analyses were performed at both time points to reflect metabolic changes initiated by rituximab therapy. Prior to treatment, several classes of glycerophospholipids were downregulated in responders relative to non-responders. Similarly, the polar 


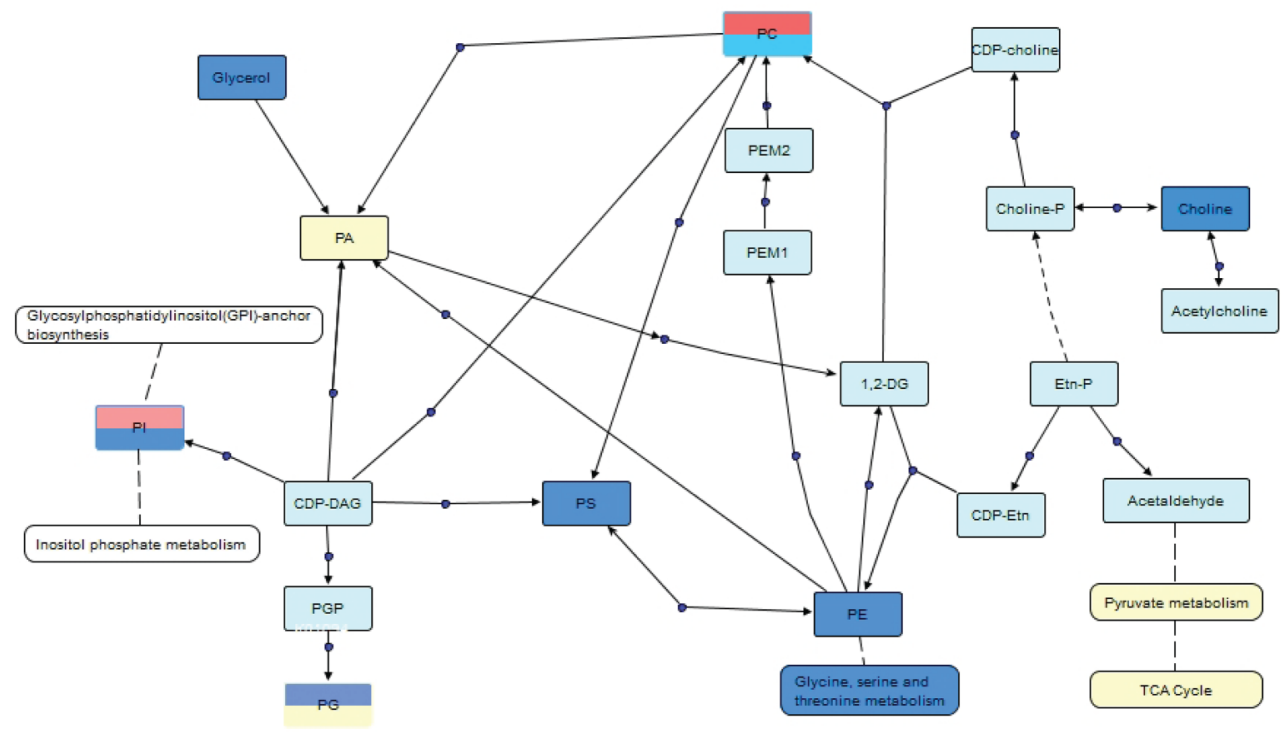

B GLYCEROPHOSPHOLIPID METABOLISM

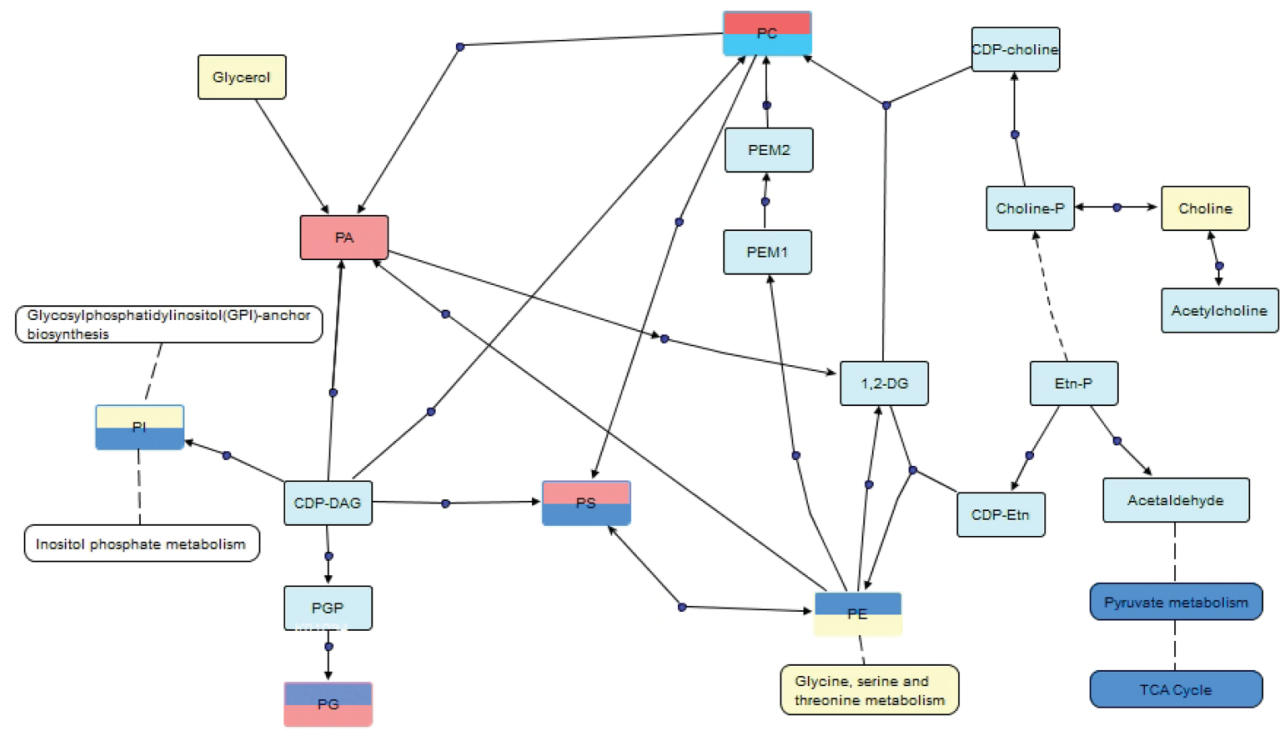

Figure 3 Glycerophospholipids and related metabolites are differentially expressed in sera from patients with RA before (A) and after (B) treatment with rituximab. Pathway map connects related classes of compounds based on biological activity. Blue indicates classes that are downregulated in responders, red indicates classes that are upregulated in responders, yellow is no significant difference and light blue indicates not detected. Split boxes represent standard ester linkage glycerophospholipids on the top and alkyl ethers on the bottom. CDP, cytidine diphosphate; CDP-Etn, CDP-ethanolamine; Etn-P, phosphoethanolamine; PA, phosphatidic acids; PC, phosphatidylcholines; PE, phosphatidylethanolamines; PEM1, phosphatidyI-Nmonomethylethanolamine 1; PEM2, phosphatidyl-N-monomethylethanolamine 2; PG, phosphatidylglycerols; PM1, phosphatidyl monomethylethanolamine; PS, phosphatidylserines; TCA, tricarboxylic acid.

metabolites related to these pathways were also lower in responders. Downregulation of the glycine, serine and threonine pathway, which is connected to PE and PS metabolism, is consistent with the observed relative intensities of these phospholipid classes in responders.
Furthermore, choline, an essential component of PC, was reduced in responders, which is consistent with the relative downregulation of O/P-PC. Free glycerol was also lower in responders when compared to nonresponders prior to treatment with rituximab. Taken 
together, these data indicate that the global metabolite signatures of responders and non-responders are distinctive before treatment with rituximab.

In many cases, treatment with rituximab either ablated or reversed the trends observed before treatment. The previously detected differences in the glycine, serine and threonine metabolic pathway as well as glycerol and choline were not observed after treatment. Instead, changes in pyruvate metabolism and tricarboxylic acid (TCA) cycle intermediates, namely, lactate, pyruvate, fumarate, citrate and succinate, contributed more significantly to differences seen between the groups following treatment. Changes in apolar metabolites involved in the glycerophospholipid pathway were consistent with their polar counterparts. Interestingly, after treatment there are distinct trends between ester and alkyl/alkenyl ether (O/P-) linkage species for all major classes of phospholipids. In fact, in many cases, opposite trends were observed between linkage types for the same class of phospholipid. These differences clearly demonstrate that global metabolite signatures of responders and nonresponders are distinctive following treatment with rituximab.

\section{DISCUSSION}

Sera collected from patients with RA were examined to identify potential biomarkers to predict response to rituximab treatment. At both time points, polar and apolar profiles were significantly different between patients with RA who were classified as either responders or non-responders according to their ACR20 scores. Prior to rituximab administration, 24 metabolites achieved $p<0.05$ between patients who would later be categorised as a responders and non-responders. When mapped into metabolic pathways, it becomes clear that metabolites involved in glycerophospholipid, amino acid and energy metabolism are all important pathways that are differentially regulated between responders and nonresponders. As expected, following rituximab therapy, there were greater differences between responders and non-responders, with 43 significant metabolites, likely due to the changes induced by rituximab treatment. Interestingly, many trends between responders and nonresponders were ablated and, in some cases, reversed the following treatment. In addition, several metabolites were significant in both groups when comparing time points. This is likely due to the fact that while both groups experienced loss of circulating B cells, there is still a discrepancy in metabolic response to rituximab as is indicated by the difference in clinical outcomes. Owing to the indiscriminate depletion of circulating B cells, some of these changes could represent the loss of metabolic pathways important within B cells and/or dependent on their presence and activation.

Currently, predictive biomarkers that would separate patient populations with RA with respect to their outcome in response to a particular therapy is an unmet need. Several approaches have been tried to address this issue. Most studies investigating differences in gene expression, mRNA and protein levels in blood have failed to successfully identify any good biomarker. ${ }^{43-45}$ Although a greater probability of clinical benefit from rituximab in CCP+ patients and normal levels of CD19+ B cells versus CCP- patient has been described, ${ }^{46}$ the effect is discreet and heterogeneous, so additional biomarkers are needed. Of interest, though, type I interferon signatures were found to negatively predict clinical response to rituximab. ${ }^{47}$ To the best of our knowledge, the metabolites that are known to be associated with interferons are in the kynurenine pathway. ${ }^{48}$ Metabolites in this pathway were either not detected or insignificant in our patient population. However, owing to the signalling potential of many lipids, in particular phosphoinositols, there may be a relationship between interferon activation and differences in lipid profiles that should be further investigated.

Here, we offer an alternative approach by identifying that global biomarker signatures may represent more appropriate approach for improving treatment protocols and outcomes of patients with RA. Metabolites serve as direct signatures of biochemical activity and may be easier to correlate with phenotype ${ }^{49}$ Perturbations in a biological system lead to correlated changes in metabolite patterns and pathways, which represent the final response to disease status or an external intervention. $^{49} 50$ NMR and MS can delineate patterns of changes and biomarkers that are highly discriminatory for the observed disease or intervention. ${ }^{1}$ A small number of metabolomics studies have been focused on identifying metabolites associated with rheumatic diseases, primarily for diagnostic purposes, ${ }^{22-24}$ but even fewer have attempted to predict response to treatment. An NMR analysis of baseline urine metabolome successfully discriminated patients with RA based on their response to anti-TNF therapy. ${ }^{2}$ Here, we used a different approach from previous studies, as we determined polar and apolar metabolite profiles by NMR and MS. Combining techniques provides more information about the pathways involved and a more complete profile to later discriminate between responders and nonresponders before treatment.

Although these findings are certainly promising, this study is not without limitations. Most importantly, we evaluated a small number of clinical samples. Despite similar clinical parameters for patient inclusion, large biological variance is expected in primary samples. Confirmation of our results with a larger sample size from an independent group of patients is necessary to strengthen our conclusions and to improve statistical confidence. In addition, analysis was performed on peripheral blood samples. However, further studies are needed to evaluate relationship between circulating metabolites and synovial pathobiology. Metabolite profiles in blood if correlating with metabolic changes in synovial tissue will certainly help to know more about 
RA aetiology. Furthermore, this study assessed response to a single drug. Future studies comparing anti-B cell, anti T-cell, anti-IL6 and anti-TNF biological treatments simultaneously to determine whether different therapies can be successfully distinguished prior to initiation of treatment have the potential to further elucidate the relationship between metabolic dysregulation and RA.

Limitations exist for each approach. In vitro experiments yield more statistically consistent data but fail to accurately recapitulate the complexity of real biological systems. Genetic and protein biomarkers also tend to be less sensitive to biological perturbations than metabolite profiles, however, they often fail to explain differences observed between individuals. For these reasons, we feel that despite the limitations of this study, we have shown promising new data for improving protocols of patients with RA by applying a metabolomics approach to differentiate response to rituximab treatment. In addition, we have laid a foundation for continued metabolomics research into RA by providing baseline metabolic profiles for future investigation into RA specific metabolism and the relationship between treatment and circulating metabolites.

Acknowledgements The authors thank Surendar Tadi for his technical support with the MS experiments.

Contributors MG and ST designed and supervised the overall project. DB and AK designed and conducted the ARISE trial. MG, ST, AK and SRS analysed the data and wrote the manuscript. SRS performed NMR and MS acquisition. SRS, AL and BW processed and interpreted NMR and MS data.

Funding This work was supported by grants from the National Institutes of Arthritis and Musculoskeletal and Skin (MG: 1K08AR064834). The support for the NMR facility was provided by the University of Texas Health Science Center at San Antonio (UTHSCSA) and National Institutes of Health Grant NCl P30 CA54174 (CTRC at UTHSCSA). Funding sources were not involved in a part of this study, including, but not limited to, data collection, analysis, reporting or publication.

Competing interests None declared.

Patient consent Obtained.

Ethics approval Patients were enrolled in the ARISE clinical trial following written informed consent. Ethical approval was granted by the Institutional Review Board (IRB) at UCSD.

Data sharing statement No additional data are available.

Open Access This is an Open Access article distributed in accordance with the Creative Commons Attribution Non Commercial (CC BY-NC 4.0) license, which permits others to distribute, remix, adapt, build upon this work noncommercially, and license their derivative works on different terms, provided the original work is properly cited and the use is non-commercial. See: http:// creativecommons.org/licenses/by-nc/4.0/

\section{REFERENCES}

1. Priori R, Scrivo R, Brandt J, et al. Metabolomics in rheumatic diseases: the potential of an emerging methodology for improved patient diagnosis, prognosis, and treatment efficacy. Autoimmun Rev 2013;12:1022-30

2. Kapoor SR, Filer A, Fitzpatrick MA, et al. Metabolic profiling predicts response to anti-tumor necrosis factor $\alpha$ therapy in patients with rheumatoid arthritis. Arthritis Rheum 2013;65:1448-56.

3. Arend WP, Firestein GS. Pre-rheumatoid arthritis: predisposition and transition to clinical synovitis. Nat Rev Rheumatol 2012;8:573-86.
4. Firestein GS. Evolving concepts of rheumatoid arthritis. Nature 2003;423:356-61.

5. Burska A, Boissinot M, Ponchel F. Cytokines as biomarkers in rheumatoid arthritis. Mediators Inflamm 2014;2014:545493.

6. Fiehn O. Metabolomics-the link between genotypes and phenotypes. Plant Mol Biol 2002;48:155-71.

7. Nicholson JK, Lindon JC. Systems biology: metabonomics. Nature 2008;455:1054-6.

8. Tiziani S, Kang Y, Choi JS, et al. Metabolomic high-content nuclear magnetic resonance-based drug screening of a kinase inhibitor library. Nat Commun 2011;2:545.

9. Guma M, Lodi A, Divakaruni A, et al. Targeting glycolysis in rheumatoid arthritis. Arthritis Rheumatol 2014;66:S416-16.

10. Guma M, Sanchez-Lopez E, Lodi A, et al. Choline kinase: a novel target for rheumatoid arthritis. Arthritis Rheum 2013;65 S542.

11. Guma M, Tiziani S, Firestein GS. Metabolomics in rheumatic diseases: desperately seeking biomarkers. Nat Rev Rheumato 2016;12:269-81.

12. Beger RD. A review of applications of metabolomics in cancer. Metabolites 2013;3:552-74.

13. Kim YS, Maruvada P, Milner JA. Metabolomics in biomarker discovery: future uses for cancer prevention. Future Oncol 2008;4:93-102.

14. Fuhrer T, Zamboni N. High-throughput discovery metabolomics. Curr Opin Biotechnol 2015;31:73-8.

15. Tiziani S, Kang $\mathrm{Y}$, Harjanto $\mathrm{R}$, et al. Metabolomics of the tumor microenvironment in pediatric acute lymphoblastic leukemia. PLoS One 2013;8:e82859.

16. Tiziani S, Lodi A, Khanim FL, et al. Metabolomic profiling of drug responses in acute myeloid leukaemia cell lines. PLoS One 2009;4: e4251.

17. Glunde K, Bhujwalla ZM, Ronen SM. Choline metabolism in malignant transformation. Nat Rev Cancer 2011;11:835-48.

18. Serkova NJ, Glunde K. Metabolomics of cancer. Tumor biomarker discovery. Springer, 2009:273-95.

19. Nagana Gowda GA, Raftery D. Can NMR solve some significant challenges in metabolomics? J Magn Reson 2015;260:144-60.

20. Cuperlović-Culf M. NMR metabolomics in cancer research. Elsevier, 2012.

21. Cuperlovic-Culf M, Culf AS, Touaibia M, et al. Targeting the latest hallmark of cancer: another attempt at 'magic bullet' drugs targeting cancers' metabolic phenotype. Future Oncol 2012;8:1315-30.

22. Kim S, Hwang J, Xuan J, et al. Global metabolite profiling of synovial fluid for the specific diagnosis of rheumatoid arthritis from other inflammatory arthritis. PloS One 2014;9:e97501.

23. Kosinska MK, Liebisch G, Lochnit G, et al. A lipidomic study of phospholipid classes and species in human synovial fluid. Arthritis Rheum 2013;65:2323-33.

24. Madsen RK, Lundstedt T, Gabrielsson J, et al. Diagnostic properties of metabolic perturbations in rheumatoid arthritis. Arthritis Res Ther 2011;13:R19.

25. Guleria A, Misra DP, Rawat A, et al. NMR-based serum metabolomics discriminates Takayasu arteritis from healthy individuals: a proof-of-principle study. J Proteome Res 2015;14:3372-81

26. Garcia-Carbonell R, Divakaruni AS, Lodi A, et al. Critical role of glucose metabolism in rheumatoid arthritis fibroblast-like synoviocytes. J Proteome Res 2016;68:1614-26.

27. Hügle $T$, Kovacs $H$, Heijnen IA et al. Synovial fluid metabolomics in different forms of arthritis assessed by nuclear magnetic resonance spectroscopy. Clin Exp Rheumatol 2012;30:240-5.

28. Kavanaugh A, Rosengren S, Lee SJ, et al. Assessment of rituximab's immunomodulatory synovial effects (ARISE trial). 1 clinical and synovial biomarker results. Ann Rheum Dis 2008;67:402-8.

29. Prevoo ML, van 't Hof MA, Kuper $\mathrm{HH}$, et al. Modified disease activity scores that include twenty-eight-joint counts. Development and validation in a prospective longitudinal study of patients with rheumatoid arthritis. Arthritis Rheum 1995;38:44-8.

30. Lodi A, Tiziani S, Khanim FL, et al. Proton NMR-based metabolite analyses of archived serial paired serum and urine samples from myeloma patients at different stages of disease activity identifies acetylcarnitine as a novel marker of active disease. PLoS One 2013;8:e56422.

31. Ludwig C, Easton JM, Lodi A, et al. Birmingham Metabolite Library: a publicly accessible database of 1-D $1 \mathrm{H}$ and 2-D $1 \mathrm{H} \mathrm{J}$-resolved NMR spectra of authentic metabolite standards (BML-NMR). Metabolomics 2012;8:8-18. 
32. Tiziani S, Einwas $\mathrm{AH}$, Lodi $\mathrm{A}$, et al. Optimized metabolite extraction from blood serum for ${ }^{1} \mathrm{H}$ nuclear magnetic resonance spectroscopy. Anal Biochem 2008;377:16-23.

33. Tiziani S, Lopes V, Günther UL. Early stage diagnosis of oral cancer using ${ }^{1} \mathrm{H}$ NMR-based metabolomics. Neoplasia 2009;11:269-79.

34. Ludwig C, Günther UL. MetaboLab-advanced NMR data processing and analysis for metabolomics. BMC Bioinformatics 2011;12:366

35. Wishart DS, Tzur D, Knox C, et al. HMDB: the Human Metabolome Database. Nucleic Acids Res 2007;35:D521-6.

36. Kanehisa M, Goto S, Sato Y, et al. Data, information, knowledge and principle: back to metabolism in KEGG. Nucleic Acids Res 2014;42: D199-205.

37. Fahy E, Subramaniam S, Murphy RC, et al. Update of the LIPID MAPS comprehensive classification system for lipids. J Lipid Res 2009;50:S9-14.

38. Cui $Q$, Lewis IA, Hegeman AD, et al. Metabolite identification via the Madison Metabolomics Consortium Database. Nat Biotechnol 2008;26:162-4

39. Smith CA, O'Maille G, Want EJ, et al. METLIN: a metabolite mass spectral database. Ther Drug Monit 2005;27:747-51.

40. Zhou B, Wang J, Ressom HW. MetaboSearch: tool for mass-based metabolite identification using multiple databases. PLoS One 2012;7:e40096.

41. Xia J, Sinelnikov IV, Han B, et al. MetaboAnalyst 3.0-making metabolomics more meaningful. Nucleic Acids Res 2015;43:W251-57.
42. Klukas $\mathrm{C}$, Schreiber F. Integration of -omics data and networks for biomedical research with VANTED. J Integr Bioinform 2010;7:112.

43. Emery P, Dörner T. Optimising treatment in rheumatoid arthritis: a review of potential biological markers of response. Ann Rheum Dis 2011;70:2063-70.

44. Charles P, Elliott MJ, Davis D, et al. Regulation of cytokines, cytokine inhibitors, and acute-phase proteins following anti-TNF-alpha therapy in rheumatoid arthritis. J Immunol 1999;163:1521-8.

45. Hueber W, Tomooka BH, Batliwalla F, et al. Blood autoantibody and cytokine profiles predict response to anti-tumor necrosis factor therapy in rheumatoid arthritis. Arthritis Res Ther 2009;11:R76.

46. Tony HP, Roll P, Mei He, et al. Combination of $\mathrm{B}$ cell biomarkers as independent predictors of response in patients with rheumatoid arthritis treated with rituximab. Clin Exp Rheumatol 2015;33:887-94.

47. Thurlings RM, Boumans M, Tekstra J, et al. Relationship between the type I interferon signature and the response to rituximab in rheumatoid arthritis patients. Arthritis Rheum 2010;62:3607-14.

48. Martin-Mola E, Balsa A, García-Vicuna R, et al. Anti-citrullinated peptide antibodies and their value for predicting responses to biologic agents: a review. Rheumatol Int 2016;36:1043-63.

49. Semerano L, Roméo PH, Boissier MC. Metabolomics for rheumatic diseases: has the time come? Ann Rheum Dis 2015;74:1325-6.

50. Patti GJ, Yanes O, Siuzdak G. Innovation: metabolomics: the apogee of the omics trilogy. Nat Rev Mol Cell Biol 2012;13:263-9. 